\title{
3d Digital Anthropometry in Case of Fit and Ergonomics of Army Uniform
}

\author{
Inga DABOLINA*, Eva LAPKOVSKA \\ Riga Technical University, Faculty of Material Science and Applied Chemistry, \\ Institute of Design Technologies, Riga, Latvia
}

DOI: $10.15221 / 18.106 \quad$ http://dx.doi.org/10.15221/18.106

\begin{abstract}
This paper focuses on analysis of human body measurements and movements - usage of laser scanning technologies for gaining measurements, movement amplitudes and biomechanical parameters in interaction within personal protective equipment (PPE). Analysis of the work postures and PPE were performed on 155 males who perform their duties in the Latvian National Armed Forces. In order to provide the comfort for individual wearers, it is essential to implement the body size correlations. The main premise is the availability of extensive and accurate information on the body measurements of certain populations or targets, as well as their distribution per body size groups or sizes. Acquisition of rapid and wide measurement data is now possible via the 3D scanning or contactless anthropometric data acquisition method. In addition to obtaining the measurements necessary for designing the clothes, the 3D technology also allows study different types of wearing habits, and the interactions between the human body and clothing layers, considering the body movement types and postures to be carried out during daily work processes, as well as the dynamics of these movements [1,2]. The right clothing and equipment is of vital importance to the survival and effectiveness of military personnel [3]. One of initial aspects is appearance and fit of uniforms and now the information necessary to ensure it can be obtained and processed using modern technologies [4]: by non-contact anthropometric methods ( $3 \mathrm{D}$ anthropometrical scanner Vitus Smart XXL $\circledast$ is used in the study), data processing automation systems, pattern making CAD/CAM systems, etc.
\end{abstract}

Keywords: 3D body scanning, anthropometrics, garment fit

\section{Introduction}

The target group of the performed research is Latvian National Armed Force (NAF) soldiers - group of 155 men. Soldiers are supplied with an army uniform for the duty wear, and the main components are the base layer jacket and trousers. The research is based on the issues that indicate supply shortages, when wearers come up with feedback about size mismatches and insufficient ergonomics. Research tasks include the identification of target-group anthropometric research capabilities, insight into uniform sizing system deficiencies, research of fit and ergonomic evaluation approaches by focusing on the use of 3D digital anthropometry to accomplish these tasks.

\subsection{Anthropometrics and sizing}

In practice, variations exist between different manufacturers' approach to compilation of sizing systems, and size coding. Access to an adequate and extensive anthropometric data is the basis for creating an appropriate sizing system. The further interpretation and operation of the data depends on the objectives of the product - what is the level of functionality, appearance, fit, and ergonomic requirements. Without the access to the target-group anthropometric data, manufacturers are forced to adapt and vary with existing databases to create sizing systems and come to a certain size range and its distribution. Usually, manufacturers aim to implement easy classification of sizes and a smaller number of sizes to be manufactured. Size charts are used for labelling garments, stock management and clothing size identification by consumers. Main tasks of the sizing are: the choice of most informative control dimensions; the justification of indifferent interval widths and implementation of scales; the calculation of the number of commercially necessary sizes; and the calculation of detailing measurements. The calculation of measurements depends on the combination of selected main dimension values, and the choice of main dimensions in parametrization is determined by the following considerations: the number of dimensions must be as low as possible, the absolute values must be as large as possible and the correlation between them must be week. Such requirements are met by total morphological features which characterize the physical development of a human - body height, chest circumference, also weight.

\footnotetext{
inga.dabolina@rtu.Iv; +371 29364004
} 
Currently, army uniform suppliers provide 56 size trousers and jackets. Sizes of a uniform jacket are based on the dimensions of the chest horizontal girth and body height, while for trousers - the waist girth and height of the body that meet the standard requirements [5]. The customer, following the size distributions and orders of the previous years, determines the orders.

However, neither the customer (NAF) nor the manufacturer has reliable information on the anthropometric data of the target group - the characteristics of the body of the soldiers, the incidence of proportions and forms, the features of physical development starting and during the service.

Individuals of the same gender and age may vary in the structure of the body and proportions of individual parts of the body, such as long legs, wide hips, long hands, short neck, and in addition, aging processes and daily physical activities may introduce changes in the body's structure. The shape of the human body depends on the level of muscle development (weak, medium, well) and the degree of fat tissue development and their relationship, as well as from the form of chest, abdomen, and back. For example, the thorax may be flat, cylindrical, conical (truncated cone); abdomen - fallen, straight, roundraised; but the back - normal, lordotic, straight. Proportionally, the largest part of the human body is the torso, on which the main garment supporting surfaces are located - shoulders and hips. In addition, the proportions of the same body height group may vary, dividing into normal, short and long leg types. Anthropological studies deal with different typological distinctions of body postures, and the identification of the main types allow usage of posture regularities in textile science. When drafting the patterns, the most convenient approach of classification is usage of the sagittal (profile) contour of the torso. It should be noted that the characteristics of soldier bodies can be particularly influenced by the physical activities throughout the service - their routine duties include carrying weapons, equipment, backpacks and various loads under different conditions - walking, running, climbing and overcoming obstacles.

Producer approach to pattern development is important when choosing ease allowances and grading intervals, as well as the type of size coding, which is essential for providing sufficient and perceptible information to consumers. The total ease allowance is formed by the physiologically minimal and movement ease allowances (including dynamic), the ease for compensation of thickness of the lower layers (package compensation), as well as the decorative-constructive or silhouette allowances. Ease allowances, which are primarily determined for the large body measurements (chest, waist, and hip circumferences), and which determine the width of the product, are necessary for both freedom of movements and breathing, with minimal pressure on the surface of the body, and providing an air layer for temperature regulation. In the selection of ease allowances, all factors relating to the body characteristics - girths, lengths, posture, shape, and proportion variables - must be taken into account. An important role in evaluating the final product is the wearing tests or fitting trials, to get end-user feedback on product compliance with fit, ergonomics and comfort.

\subsection{Body movements}

Soldiers carry out various body movements in their daily tasks, which may create tensions in different clothing zones, but should not affect the freedom of movements or cause mechanical forces that can result in pressure on the body parts or abrasions on the skin. Daily body movements of soldiers include shooting, deep lunge and squat postures, and crawling, rolling, holding and lifting heavy equipment, and different climbing movements.

Human movements are very complicated, since the movement apparatus is a mechanical system consisting of more than 200 bones and several hundred tendons. The total number of movements in the joints (so-called free movements) exceeds 250 types, but the number of muscles that serve movements exceeds 600 . The human movement is mechanical, i.e. it is a change in the condition of a moving body or parts of it relative to other bodies [6]. A complex muscular system is responsible for the posture and movements of the human body. For example, pectoral girdle is defined as the structure which attaches the upper limbs to the axial skeleton, namely the clavicles and scapulae, to which the muscles of the pectoral girdle attach; however, there are few muscles which connect the upper limb directly to the central skeleton and cause indirect movements of the pectoral girdle. Muscles of the trunk include the muscles involved in breathing - muscles of respiration [7]. When breathing in - the lower ribs are lifted up and out to the sides in what had been described as a 'bucket handle' movement that increases the width of the ribcage. The sternum (breastbone) also moves out, further expanding the chest. [8].

The direction of anthropometric research, the aim of which is to investigate body surface changes during movements, is the anthropometric dynamics. Investigations are made of the most commonly occurring movements or postures, making measurements at the maximum possible amplitude. If there are known coordinates of the human body points or landmarks, it is possible to describe its position in room. Therefore, in order to describe the position of the human body, anatomy introduces the concepts of the 
human body planes (frontal, sagittal, transverse) and axes [6]. The advantage of 3D scanning is the use of physical markers to fix anthropometric landmarks on the human body. However, in the case of individual postures, the use of 3D scanners is limited by the permissible scanning range - for example, the scanner used in this study $-1100 \mathrm{~mm}$ in width, $1000 \mathrm{~mm}$ in depth and $2100 \mathrm{~mm}$ in height.

Dynamic indicators are obtained for the largest body measurements that determine the dimensions of the product - for example, the width of the back (in relation to the chest circumference), the length of the arms, the length of the legs etc. For example, in studies, the length of the back, leaning forward, shows an average increase of $+15.8 \mathrm{~cm}$ for men, a length of the leg in a climbing movement of +5.5 $\mathrm{cm}$, and a knee circumference of $3.3 \mathrm{~cm}$ [9]. Increases or decreases of body measurements are used in integrating them into ease allowances or constructive-technological solutions.

\section{Materials and methods}

The 3D anthropometric scanner Vitus Smart XXL® (@ Human Solutions GmbH and VITRONIC GmbH) with the AnthroScan data processing system is used for soldier body measurements, as well as manual measurement tools for control measurements - measure tape and anthropometer. All measurements are gained by professionals with appropriate knowledge and skills in anthropometry. The findings of the 3D scan advantages in obtaining anthropometric data, the differences from manual methods and 3D scanning applications in problem analysis are reflected, taking into account also previous studies.

Analysis of the field's uniforms jackets structural parameters is carried out for the analysis of the conformity of uniform sizing system used in production. Identification of the horizontally dimensional chest and package compensation is performed.

Dynamic postures are scanned, which can partly characterize the daily movements of soldiers. In separate experiments, physical markers are used for fixing anthropometric landmarks on the body of the test person, as well as on the product (jacket) with the ability to measure marker relocations.

For the fit assessments scanning of an undressed and dressed body is carried out to superimpose scans - both in the standard upright position and in dynamic poses.

Attempts are made to fix the dynamic body postures to ensure their repeatability, using additional means for marking the hand positions.

AnthroScan tools are used in different stages of the study to obtain transverse, frontal and sagittal cross-sections. Cross-sections of an undressed body, dressed body and their superimpositions are gained.

\section{Tests and results}

During the scanning process and interviews of soldiers about uniform sizing flaws, certain groups of wearers were observed with difficulties choosing the uniform jackets of appropriate size. Chest girths in manufacturer size tables are divided into indifferent intervals ( 8 in total) with a width of $8 \mathrm{~cm}$. It has been identified that the horizontal dimensional chest and package compensation for all sizes is $+28 \mathrm{~cm}$. In the cases when the measurement of the soldier chest girth reaches the end margin of the interval, fit is recognized as unsatisfactory, while already choosing the next (bigger) size jacket, it is too large taking into account the increase of the interval.

The ease allowance or compensation is adequate, also relying on the recommendations in the literature on the amount of allowances, for example, $18-34 \mathrm{~cm}$ for the chest circumference is sufficient (lightweight fabric $+18 \mathrm{~cm}$, medium $+24 \mathrm{~cm}$, heavy $+28 \mathrm{~cm}$ ) [9]. However, the situation with such groups of soldiers calls into question the choice of the width of the chest girth intervals, which is not satisfactory for individuals at the margins of the intervals. It is obvious that the manufacturers are not interested in increasing the number of sizes by reducing the width of the interval, but only by obtaining and analysing data of the entire target group it is possible to determine the total amount of required sizes. Therefore, it is necessary to obtain a broad anthropometric data of target group in order to review or create a new distribution of sizes with appropriate indifferent interval widths, since the existing mix of different systems is not valid within a part of particular target group.

Moreover, it should be noted that according to previous researches weaknesses in the current approach of measuring soldier chest girth are noticed. Measurements are obtained by manual methods, but studies show that the resulting chest girth may not be really horizontal and is affected by the direction of the torso axis. It can be critical in the selection of appropriate size jacket because both in the sizing systems and in most pattern construction methods, the horizontal chest circumference is used.

The scans of soldiers group (problematic) with a chest circumference of $106 \mathrm{~cm}( \pm 1 \mathrm{~cm})$ and a body height of $173 \mathrm{~cm}( \pm 2 \mathrm{~cm})$ are summarized in figure 1 . 

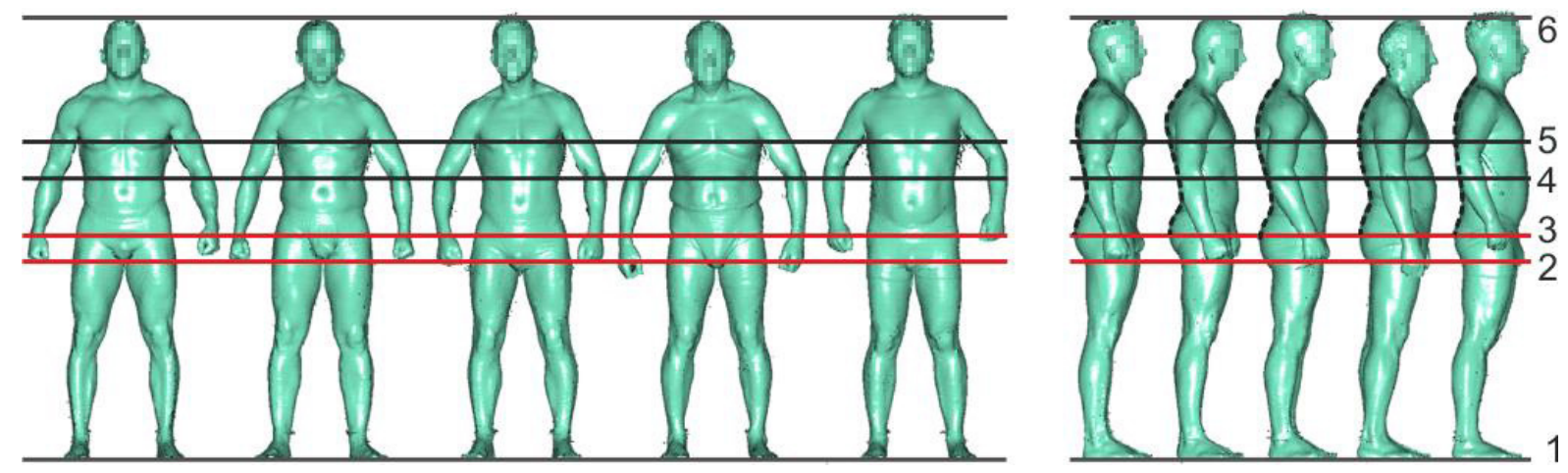

Fig. 1. Scans of soldier group - evaluation of stature and proportions (horizontal chest girth $106 \mathrm{~cm}$ )

Use of the 3D scanning additionally allows to evaluate the stature and proportions of test persons (see figure 1), as well as cross-sections are usable to compare individual parts of the body of individuals, such as figure 2. where horizontal chest girth level cross-sections of specific group are superimposed for comparison.

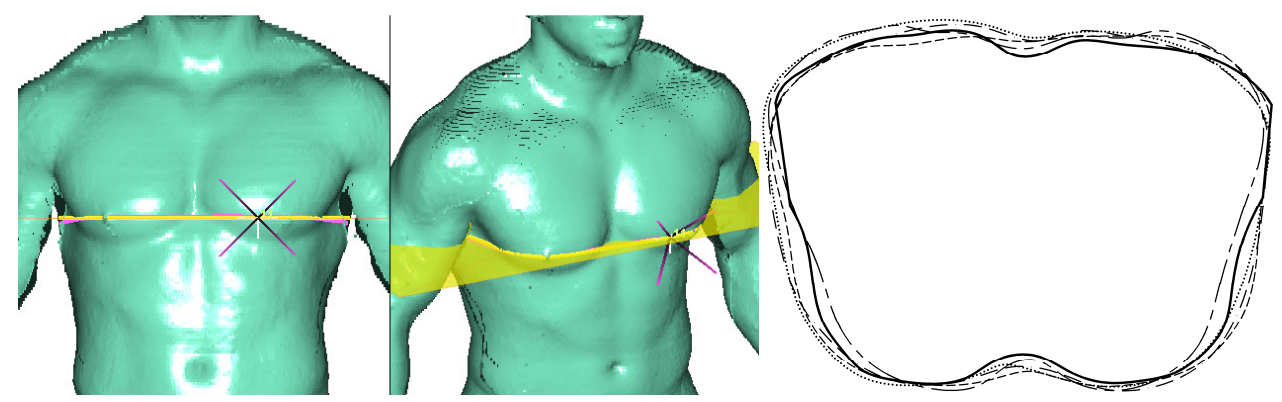

Fig. 2. Comparison of horizontal chest girth cross-sections (horizontal chest girth $106 \mathrm{~cm}$ )

Observations on wearing habits regarding the trousers have shown that they are not worn at the anthropometric waist level but at the waistband level. By introducing changes to the trouser patterns (lowering the waist height) there must be reliable data on the measurements of the wearing level. 3D system automatically retrieves the waistband circumference, in addition, its shape is not measured parallel to the plantar plane (as it is for anthropometric waist level) but is oblique. Comparison of waist and waistband level shapes by extracting transversal cross-sections is shown in figure 3.
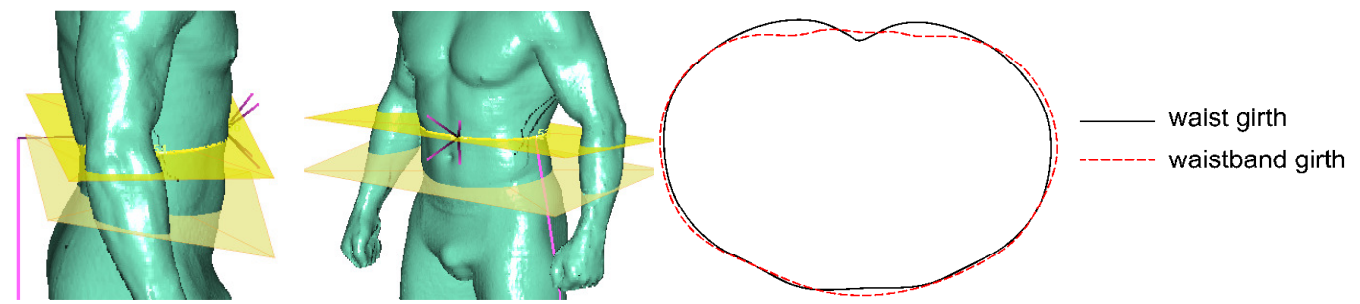

Fig. 3. Comparison of waist girth and waistband girth level cross-sections

The differences between the crotch length and shape depending on the measurement to the waist or waistband level are shown in figure 4. By analysing body shapes in this way, appropriate changes can be made in the proportions of the front and back of the trouser patterns regarding the crotch line.
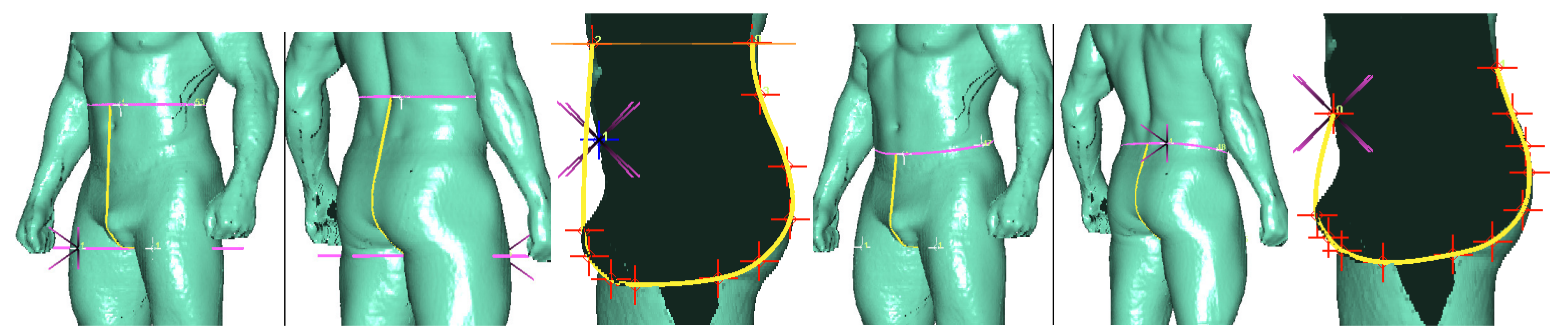

Fig. 4. Crotch lengths from waist girth and waistband girth levels 
At present, body height is used as the second dimension for trouser sizing. Comparison of proportions in figure 1 shows that people of one body height may have different leg lengths that can affect fit when choosing one size trousers. The sizing system should also be reviewed considering the possibility of easy and fast data acquisition on the inseam measurements by 3D scanning.

Scanning of dynamic poses allows to obtain indicators of significant changes in body measurements. The disadvantages are that some areas that are not reached by the laser beam are not measurable which in dynamic studies can be important for separate body parts, for example, it would be necessary to get measurements of crotch area in the squat position. Examples of measurements gained from dynamic postures are shown in figure 5 when planes are set for defining direction of measurements and manual measurements performed on the surface of the body scan. The advantage of the system is that it is possible to carry out additional programming to introduce new body measurements. Comparing with the stature posture measurement of the back width the difference of $18,9 \mathrm{~cm}$ of was shown in the bending forward position.

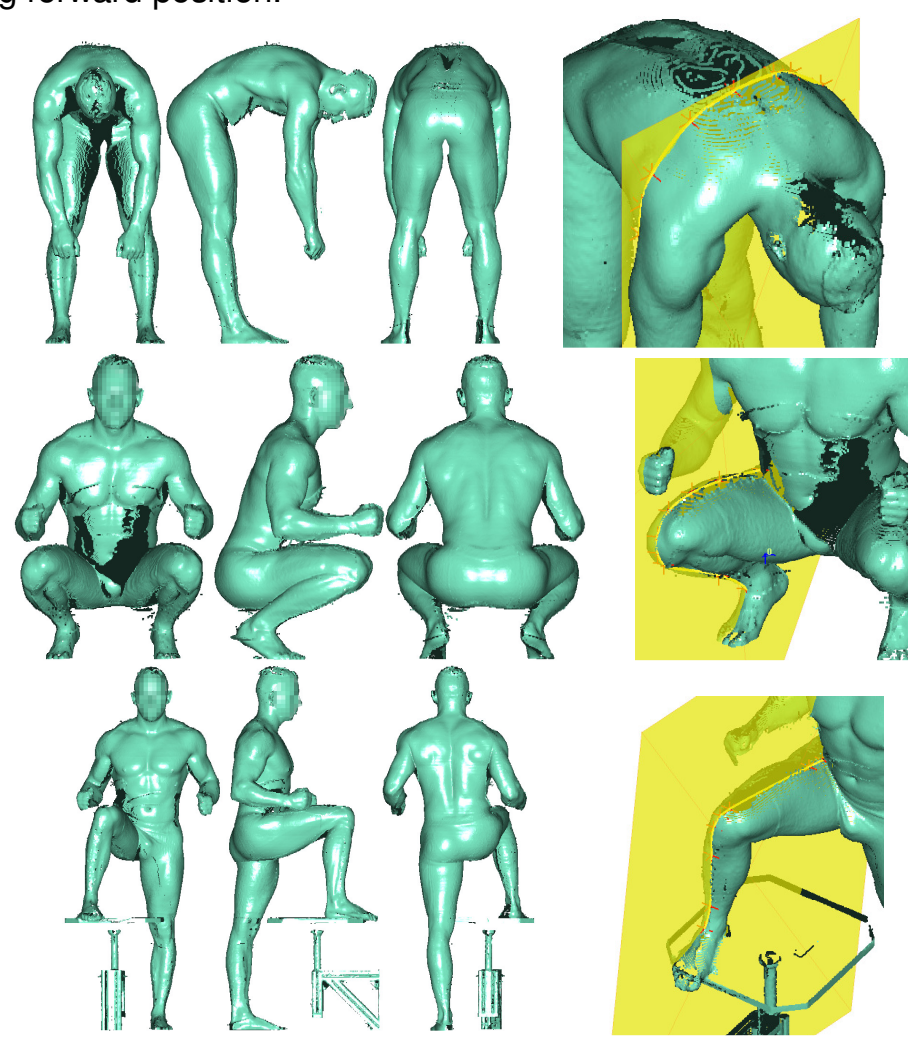

Fig. 5. Measurements in dynamic postures

For evaluation of appearance and fit of clothing dressed body scans can be obtained - both in static and dynamic poses, which can serve as a kind of work movement imitation. Static and dynamic postures of dressed body for evaluation of uniform jacket are shown in figure 6. Assistive objects with special marks for the placement of body parts can be used for posture fixation (tube in figure 6 for palm location) ensuring options of accurate superimpose with undressed body. It has been observed that this also does not guarantee that there will be no changes in individual parts of the body considering the human fatigue level, breathing movements and any other posture changes.
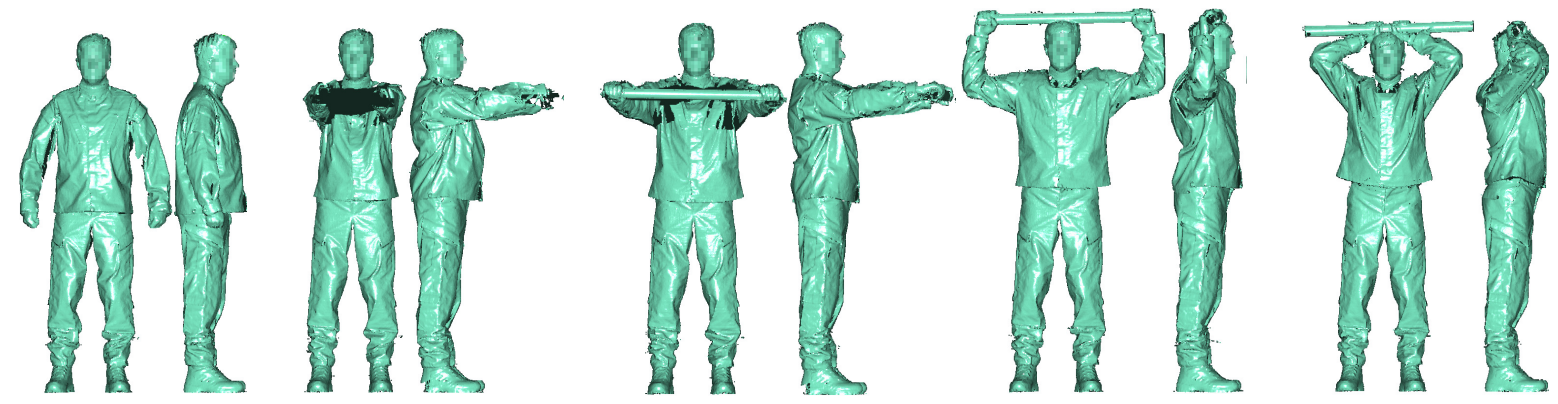

Fig. 6. Dressed body postures for evaluation of the uniform appearance 
The results reflected in the research demonstrate that the 3D scanned image clearly displays the fit and appearance of the sample. On the scanned surface the defect wrinkles and other imperfections, if any, are clearly visible, but the use of superimpose options and cross-sections allows getting an idea regarding the sample volume in respect of a human body (see figure 7).

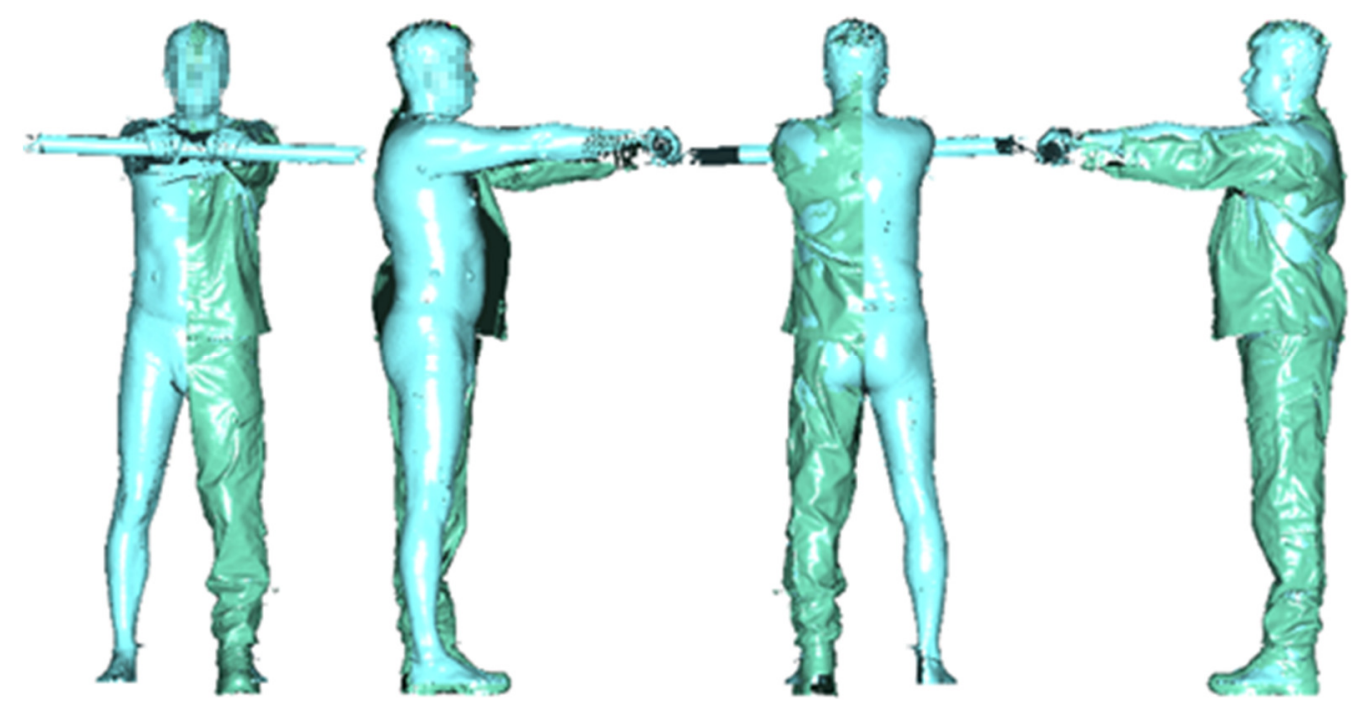

Fig. 7. Evaluation of the uniform appearance

The use of physical markers (demonstrated in figure 8.) makes it possible to measure the dislocation of clothing along the surface of the body or changing parts of the garments during movement, superimposing scans of static and dynamic postures.

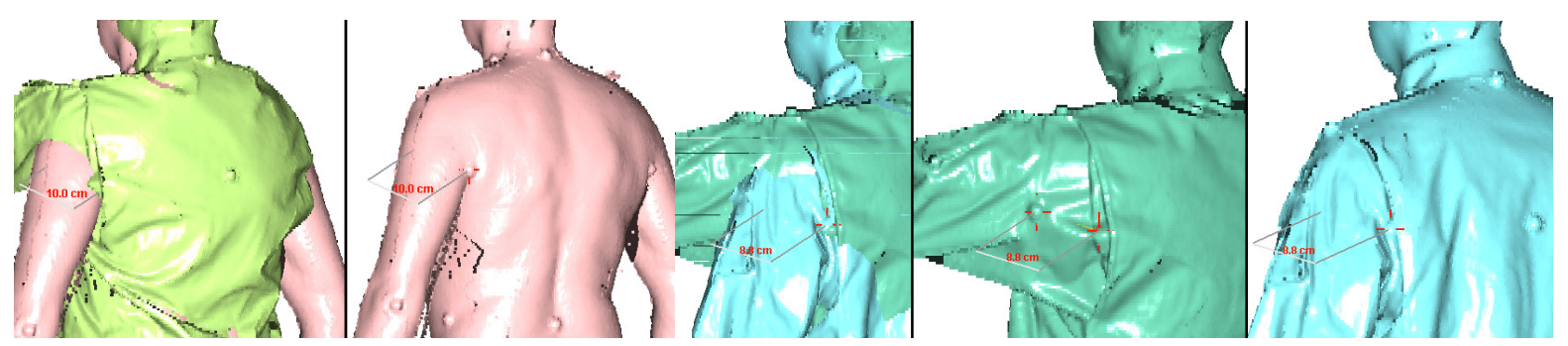

Fig. 8. Evaluation of the uniform appearance by distance measurements between physical markers

The cross-sections obtained by superimposing dressed and undressed bodies and by applying additional vector graphic systems allows to measure and analyse of a gap between the body and clothing layer. The volume of the ease allowance can be regarded as the difference between lengths of the outer and inner circles or contours. If the contour is closed, an area between the body and the clothing layer can be subjected to the analysis and the distance measurements allow to judge the fit in different body areas. Transversal cross-sections of chest level (above), waist level and hip level with distance measurements are shown in figure 9 .
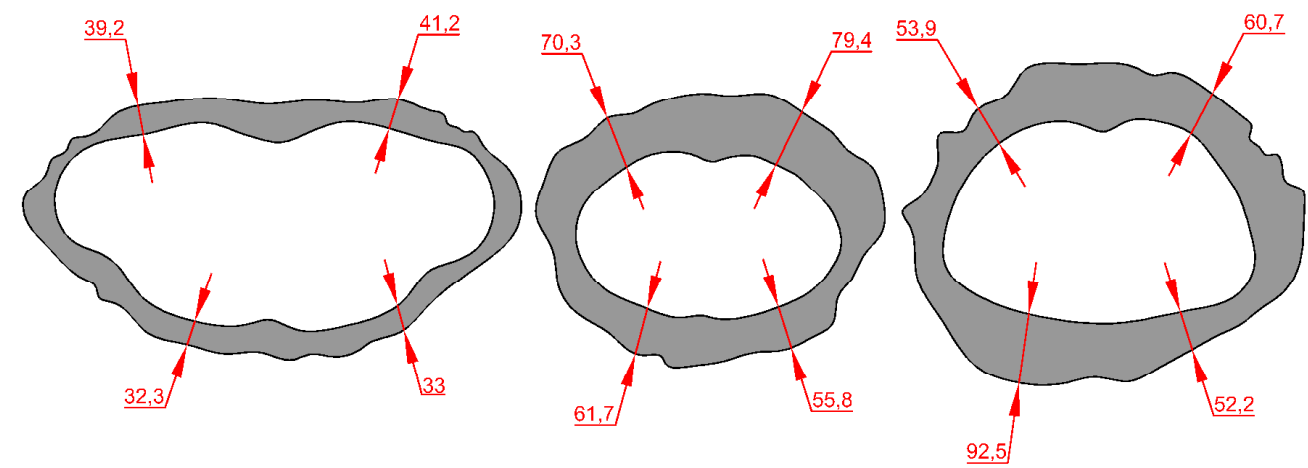

Fig. 9. A complex evaluation of the uniform appearance 


\section{Conclusions}

Creation of end-user satisfactory garment sizing and coding system is not possible without adequate anthropometric data. At best, anthropometric data are obtained of a particular target group by creating a sizing system that meets the product's purpose, taking into account both the expected functionality level and the aspects of user experience and needs. An advantage of 3D scanning for these purposes is the fast, non-contact acquisition of reliable data of big groups of people, with the options of reproducible measurements and additional research.

There is still no common approach or standards for fit and ergonomic assessment, considering the influences of many different factors depending on the type of the product and the wearing conditions. Therefore, fit and ergonomic assessment should be a complex approach, both in wearing tests obtaining the opinion of the wearer and understanding their needs and, accordingly, looking for a correlation with the features of body measurements within the target group and the measurements of the finished products in relation to the human body. Information about end-user wearing habits may also play an important role in the design development. 3D scanning can be used for this purpose, obtaining scans of finished products and analysing their volumes, fit, and interactions with the human body. As all the samples have been evaluated as having adequate fit and appearance, the usefulness of such automated data reading systems may be considered appropriate and the anthropometric data gathered by 3D scanner usable in automatically designed pattern blocks by CAD/CAM system.

Anthropometric data acquisition is critical for sizing system development by choosing control dimensions for sizing, by introducing indifferent intervals and the total number of sizes. Also, the availability of indicators of the target group physical development and body form types is needed. Already at the stage of ease allowance selection the knowledge of the body movements (dynamics) that are performed during everyday duties is essential. Different 3D scanning tools are usable for analyse of target group body types and dynamic indicators.

3D scanning has a number of advantages over manual methods such as speed, convenience, reliability and reproducibility of results, and the presence of tools, such as the acquisition of cross-sections, which is practically impossible with manual methods. Still, development is evident in the acquisition of more complete scans without invisible zones, as well as in ensuring the repeatability of the poses required for precise superimpose.

\section{ACKNOWLEDGMENT}

This research work was partially financed by the European Union's European Regional Development Fund, through the INTERREG BSR Programme, which awarded a grant to the SWW project (\#R006). The authors gratefully acknowledge the received financial support.

\section{References}

[1] "Anthropometry, apparel sizing and design", Woodhead Publishing Series in Textiles: Number 148, United Kingdom, 2014, 4-26

[2] R. F. Goldman, Bernhard Kampmann, "Handbook on Clothing", 2nd Edition, 2007, Chapter 17, 712.

[3] E. Sparks "Advances in Military Textiles and Personal Equipment" ISBN: 9781845696993, Woodhead Publishing, 2012

[4] J.C.Tsan-Ming "Information Systems for the Fashion and Apparel Industry" ISBN: 978-0-08100571-2, Elsevier, 2016

[5] EN ISO 13688:2013 Protective clothing - General requirements

[6] Г. И. Попов "Биомеханика", Академия, 2005, ISBN: 5-7695-2121-X

[7] B. J. Cohen, K. Hull, "Memmler's structure and function of the human body", 11th edition, Wolters Kluwer, 2016

[8] "The human body", An illustrated guide to your body and how it works, ed P. Abrahams, London: Amber Books Ltd, 2017, 68-103

[9] Э. Амирова, О. Сакулина, “Изготовление специальной и спортивной одежды”, Легкая промышленность и бытовое обслуживание, 1985 